\title{
Serotypes and antimicrobial resistance profiles of Salmonella enterica recovered from clinical swine samples
}

\author{
Siriporn Kongsoi ${ }^{1}$, Suksun Chumsing ${ }^{1}$ (D), Darunee Satorn² ${ }^{(D)}$ and Panisa Noourai² \\ 1. Department of Veterinary Public Health, Faculty of Veterinary Medicine, Kasetsart University, Nakhon Pathom 73140, \\ Thailand; 2. Department of Microbiology, Faculty of Public Health, Mahidol University, Bangkok 10400, Thailand. \\ Corresponding author: Siriporn Kongsoi, e-mail: fvetsks@ku.ac.th \\ Co-authors: SC: fvetsuc@ku.ac.th, DS: darunee_satorn@hotmail.com,PN: panisa595@gmail.com \\ Received: 12-06-2020, Accepted: 22-09-2020, Published online: 03-11-2020
}

doi: www.doi.org/10.14202/vetworld.2020.2312-2318 How to cite this article: Kongsoi S, Chumsing S, Satorn D, Noourai P (2020) Serotypes and antimicrobial resistance profiles of Salmonella enterica recovered from clinical swine samples, Veterinary World, 13(11): 2312-2318.

\begin{abstract}
Background and Aim: Salmonella enterica is an important foodborne pathogen and is recognized as a major public health issue. The emergence of multidrug-resistant (MDR) S. enterica represents a major challenge for national public health authorities. We investigated the distribution of serovars and antimicrobial resistance of $S$. enterica isolates from clinical swine samples stored at the Veterinary Diagnostic Laboratory, Faculty of Veterinary Medicine, Kasetsart University from 2016 to 2017.

Materials and Methods: Clinical samples were collected and subjected to standard microbiological techniques outlined in the Manual of Clinical Microbiology to identify Salmonella serovars. Susceptibility to antimicrobials was tested by the Kirby-Bauer disk diffusion method using a panel of 14 antimicrobials.

Results: A total of 144 Salmonella isolates were identified and the dominant serovar was Salmonella Choleraesuis (66.67\%), followed by monophasic Salmonella Typhimurium (18.75\%), S. Typhimurium (9.03\%), and Rissen (5.56\%). The isolates displayed high resistance rates to ampicillin (AMP [100\%]), amoxicillin (AX [100\%]), tetracycline (TE [100\%]), cefotaxime (CTX [89.58\%]), ceftriaxone (CRO [87.50\%]), chloramphenicol (C [82.64\%]), gentamicin (CN [79.17\%]), nalidixic acid (NA [72.92\%]), and ceftazidime (CAZ [71.53\%]). All isolates were MDR, with 29 distinct resistance patterns. The dominant MDR pattern among serovars Choleraesuis and Rissen exhibited resistance to 9 antimicrobials: (R7-14 AMP-AX-CAZ-CRO-CTX-NA-C-CN-TE). However, all tested isolates were susceptible to AX/ clavulanic acid and fosfomycin.
\end{abstract}

Conclusion: High resistance levels to the third generation of cephalosporins such as CAZ, CRO, and CTX highlight the need for careful and reasonable usage of antimicrobials in animals and humans, especially for $S$. Choleraesuis infections.

Keywords: clinical isolates, multidrug resistance, Salmonella Choleraesuis.

\section{Introduction}

Salmonella enterica is a major foodborne zoonotic agent associated with industrially produced food animals and is thus a public health concern in many countries [1]. S. enterica is one of the important enteric pathogens in swine, causing enterocolitis and/ or acute septicemia in animals of all ages, particularly in weaned and nursery swine [2-4]. Symptoms of salmonellosis in swine vary widely from reduced weight gain to sudden death, all of which translate to economic losses in the swine industry worldwide [2-5].

Swine are considered an important reservoir host for many serovars of Salmonella and contaminated pork is a known source of human infections [6,7]. Antimicrobials have been used in swine for

Copyright: Kongsoi, et al. Open Access. This article is distributed under the terms of the Creative Commons Attribution 4.0 International License (http://creativecommons.org/licenses/ by/4.0/), which permits unrestricted use, distribution, and reproduction in any medium, provided you give appropriate credit to the original author(s) and the source, provide a link to the Creative Commons license, and indicate if changes were made. The Creative Commons Public Domain Dedication waiver (http:// creativecommons.org/publicdomain/zero/1.0/) applies to the data made available in this article, unless otherwise stated. therapeutic purposes to treat disease, for prophylactic or metaphylactic purposes to prevent disease, and for growth promotion [8]. Due to the overuse of antimicrobials in recent years, the rate of resistant and even multidrug-resistant (MDR) Salmonella has risen dramatically, resulting in more frequent failures in the treatment of human salmonellosis [9-12]. The potential for treatment failures, especially in life-threatening situations, as a result of antimicrobial-resistant Salmonella infections highlights the need for further research and surveillance targeting Salmonella in the pork production chain. In particular, information on the distribution of antimicrobial resistance in S. enterica serovars among healthy and clinically affected swine is important to public health. Several studies have investigated the incidence of antimicrobial-resistant Salmonella carriage in apparently healthy swine from finishing herds [13-15]. However, there has been relatively limited research on the serotype diversity of resistant $S$. enterica isolates recovered from clinically diseased swine. As mentioned above, there is a need for more information on the distribution of antimicrobial resistance in $S$. enterica serovars among healthy 
and clinically affected swine. Clinical isolates are especially important for antimicrobial resistance monitoring and surveillance efforts because clinically ill animals receive more antimicrobial treatments; therefore, these clinical isolates have potentially encountered the most antimicrobial selective pressure.

The objectives of this study were to examine the serovar distribution and antimicrobial resistance among Salmonella isolates recovered from various clinical samples of swine submitted to the Veterinary Diagnostic Laboratory, Faculty of Veterinary Medicine, Kasetsart University, Thailand. Our approach can be the basis for developing an early warning system for emerging pathogens and antimicrobial resistance threats. Our results will provide important information to develop management strategies and policy decisions on the monitoring and surveillance of Salmonella in food animals.

\section{Materials and Methods}

\section{Ethical approval}

Ethical approval was not necessary for this study.

\section{Study location, period and sample collection}

The Veterinary Diagnostic Laboratory, Faculty of Veterinary Medicine, Kasetsart University receives clinical samples (whole animals, carcasses, swabs, tissues, and organs) predominantly from swine farms within Nakhon Pathom and Ratchaburi, the two largest pig-producing provinces in Thailand. All clinical samples originally collected by postmortem examination (necropsy) and delivered to the laboratory for diagnostic purposes, and thus no experimental investigation on swine was performed. All swine clinical samples from July 2016 and through August 2017 were included in this study.

\section{Isolation and Identification}

Clinical samples consisted of materials submitted for isolating and identifying Salmonella in the course of routine diagnostic procedures. The clinical samples were directly plated onto MacConkey agar (Oxoid, UK) and xylose lysine deoxycholate (Oxoid, UK) agar and incubated for $24 \mathrm{~h}$ at $37^{\circ} \mathrm{C}$. The plates were examined for typical Salmonella colonies and up to five presumptive Salmonella colonies were selected and identified biochemically. Various biochemical media were inoculated and incubated overnight at $37^{\circ} \mathrm{C}$, and the results were observed and interpreted [16].

\section{Serotyping}

The serogroup of each isolate was identified using BD Difco ${ }^{\mathrm{TM}}$ Salmonella $\mathrm{O}$ antisera (Becton Dickinson, US) at the Veterinary Diagnostic Laboratory, Nakhon Pathom. The serotype was determined according to the White-Kauffmann-Le Minor scheme [17,18], which involves slide agglutination to define $\mathrm{O}$ and $\mathrm{H}$ antigens using commercial antisera obtained from the S\&A Reagents Lab, Bangkok.

\section{Antimicrobial susceptibility testing}

The antimicrobial susceptibilities of Salmonella isolates to 14 types of antimicrobials were determined by the standard Kirby-Bauer disk diffusion method according to the guidelines and interpretative criteria of the Clinical and Laboratory Standards Institute (CLSI, 2017) [19]. The following antimicrobial disks (Oxoid, UK) were used in this study: Ampicillin (AMP, $10 \mu \mathrm{g}$ ), amoxicillin (AX, $25 \mu \mathrm{g}$ ), AX/clavulanic acid (AMC, 20/10 $\mu \mathrm{g}$ ), cefotaxime (CTX, $30 \mu \mathrm{g}$ ), ceftriaxone (CRO, 30 $\mu \mathrm{g})$, ceftazidime (CAZ, $30 \mu \mathrm{g})$, gentamicin $(\mathrm{CN}, 10$ $\mu \mathrm{g})$, tetracycline (TE, $30 \mu \mathrm{g})$, chloramphenicol (C, $30 \mu \mathrm{g}$ ), nalidixic acid (NA, $30 \mu \mathrm{g}$ ), ciprofloxacin (CIP, $5 \mu \mathrm{g}$ ), trimethoprim/sulfamethoxazole (SXT, $1.25 / 23.75 \mu \mathrm{g}$ ), colistin (CT, $10 \mu \mathrm{g}$ ), and fosfomycin (FOT, $200 \mu \mathrm{g})$. The reference strain, Escherichia coli ATCC 25922, was used as a control. According to the CLSI, each isolate was evaluated as either susceptible, intermediate, or resistant. In addition, Salmonella isolate that is resistant to at least three antimicrobial classes was defined as an MDR Salmonella isolate.

\section{Results}

\section{Prevalence and serovar distribution}

We processed a total of 337 clinical samples from 163 pigs in 46 farms, yielding a total of 144 Salmonella isolates that were recovered and identified from $52(15.43 \%)$ clinical samples. The isolation rates of Salmonella were $77.78 \%$ (7/9) in spleen samples, $50.00 \%$ (14/28) in mesenteric lymph node (MSLN) samples, 14.29\% (20/140) in lung samples, $13.33 \%(6 / 45)$ in intestine samples, $8.33 \%(1 / 12)$ in brain swab samples, $4.65 \%(2 / 43)$ in joint swab samples, $3.45 \%(1 / 29)$ in tracheobronchial lymph node (TBLN) samples, and $3.23 \%(1 / 31)$ in tonsil samples.

We identified four serovars: Salmonella Choleraesuis $(96 / 144,66.67 \%)$ was the most predominant, followed by monophasic Salmonella Typhimurium (27/144, 18.75\%), S. Typhimurium (13/144, 9.03\%), and $S$. Rissen (8/144, 5.56\%). The distribution of these serovars among the

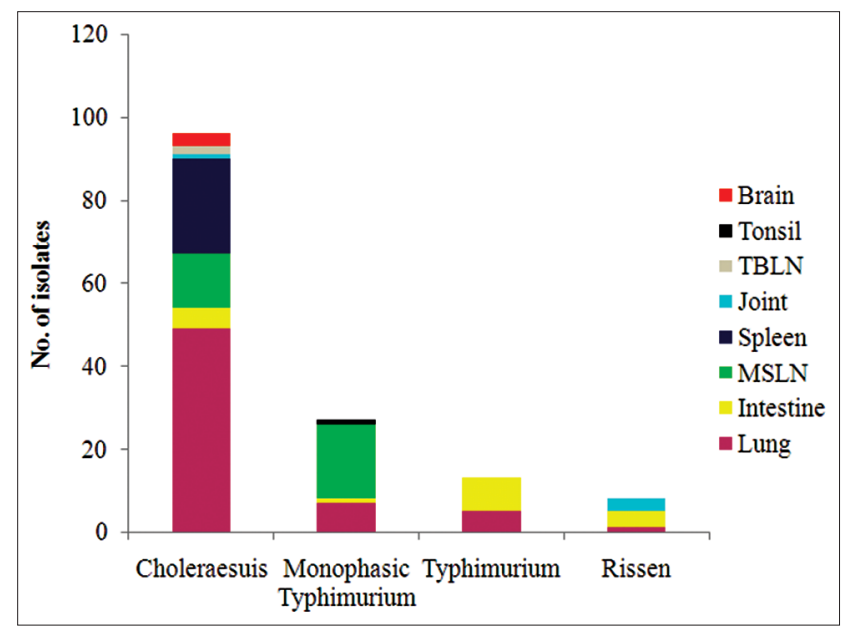

Figure-1: The distribution of Salmonella serovars in various clinical samples. 
different clinical samples is given in Figure-1. Serovar Choleraesuis was most prevalent in lung samples $(49 / 96,51.04 \%)$, followed by spleen samples $(23 / 96$, 23.96\%), MSLN samples (13/96, 13.54\%), intestine samples $(5 / 96,5.21 \%)$, brain swab samples $(3 / 96$, $3.13 \%)$, TBLN samples $(2 / 96,2.08 \%)$, and joint samples $(1 / 96,1.04 \%)$.

Serovar monophasic Typhimurium was isolated from MSLN (18/27, 66.67\%), lung (7/27, 25.93\%), intestine $(1 / 27,3.70 \%)$, and tonsil $(1 / 27,3.70 \%)$ samples. Serovar Typhimurium was detected in intestine $(8 / 13,61.54 \%)$ and lung $(5 / 13,38.46 \%)$ samples, while serovar Rissen was isolated from intestine $(4 / 8$, $50.00 \%)$, joint swab $(3 / 8,37.50 \%)$, and lung $(1 / 8$, $12.50 \%$ ) samples.

\section{Antimicrobial resistance and multidrug resistance patterns}

Antimicrobial susceptibility testing of the 144 isolates showed that they were all sensitive to AMC acid and FOT; and they were all resistant to AMP, AX, and TX. The following antibiotics also elicited resistance among the isolates (arranged in decreasing frequency of resistant isolates): Cefotaxime $(129,89.58 \%)$, followed by ceftriaxone (126 isolates, $87.50 \%)$, chloramphenicol (119, 82.64\%), gentamicin $(114,79.17 \%)$, nalidixic acid $(105$, $72.92 \%)$, ceftazidime $(103,71.53 \%)$, trimetho$\mathrm{prim} / \mathrm{sulfamethoxazole}(21,14.58 \%)$, colistin $(20$, $13.89 \%)$, and ciprofloxacin $(15,10.42 \%)$. In addition, the following antibiotics elicited intermediate resistance among the isolates: Ciprofloxacin $(112,77.78 \%)$, trimethoprim/sulfamethoxazole (52, $36.11 \%)$, ceftazidime $(19,13.19 \%)$, nalidixic acid
$(7,4.68 \%)$, and ceftriaxone $(3,2.08 \%)$. Furthermore, the isolates were most susceptible to colistin (124, $86.11 \%$ ), followed by trimethoprim/sulfamethoxazole $(71,49.31 \%)$, nalidixic acid $(32,22.22 \%)$, gentamicin (30, 20.83\%), chloramphenicol (25, $17.36 \%)$, ceftazidime $(22,15.28 \%)$, ciprofloxacin $(17,11.81 \%)$, ceftriaxone $(15,10.42 \%)$, and cefotaxime $(15,10.42 \%)$.

The prevalence levels of antibiotic resistance among Salmonella serovars are shown in Figure-2. For Choleraesuis isolates, 100\% (96/96) were resistant to AMP, AX, TE, CTX, and NA; 97.92\% (94/96) were resistant to CRO; $89.58 \%$ (86/96) were resistant to $\mathrm{C} ; 83.33 \%(80 / 96)$ were resistant to $\mathrm{CAZ}$ and $\mathrm{CN} ; 15.63 \%$ (15/96) were resistant to SXT; $14.58 \%$ (14/96) were resistant to CIP; and $10.42 \%(10 / 96)$ were resistant to CT.

For the monophasic serovar, $100 \%(27 / 27)$ were resistant to AMP, AX, and TE; 51.85\% (14/27) were resistant to $\mathrm{CN}$; $44.44 \%$ (12/27) were resistant to CTX and $\mathrm{C} ; 40.74 \%(11 / 27)$ were resistant to CRO; $22.22 \%$ (6/27) were resistant to SXT and CT; $18.52 \%(5 / 27)$ were resistant to CAZ; and $3.70 \%(1 / 27)$ were resistant to NA.

For the Typhimurium isolates, $100 \%(13 / 13)$ were resistant to AMP, AX, TE, CRO, CTX, and C; $92.31 \%(12 / 13)$ were resistant to $\mathrm{CN} ; 84.62 \%(11 / 13)$ were resistant to CAZ; and $30.77 \%$ (4/13) were resistant to CT.

For the Rissen isolates, $100 \%(8 / 8)$ were resistant to AMP, AX, TE, CRO, CTX, NA, C, and CN; $87.50 \%(7 / 8)$ were resistant to CAZ; and $12.5 \%(1 / 8)$ were resistant to CIP.

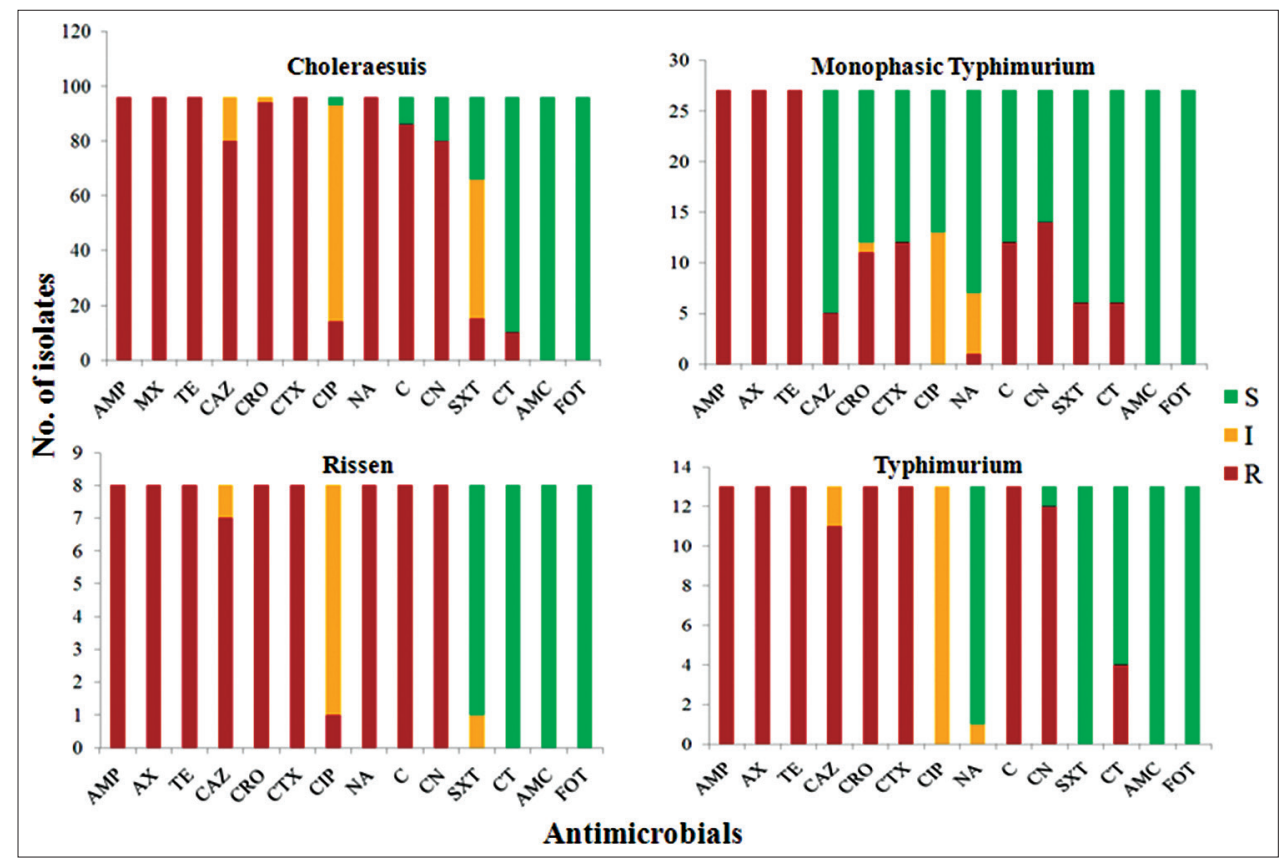

Figure-2: Antimicrobial resistance diversity of each Salmonella serovar. Red indicates resistance (R) to the corresponding antimicrobials, yellow indicates intermediate (I) resistance, and green indicates susceptible (S) phenotype. The names of the antimicrobials are abbreviated as ampicillin (AMP), amoxicillin (AX), tetracycline (TE), ceftazidime (CAZ), ceftriaxone (CRO), cefotaxime (CTX), ciprofloxacin (CIP), nalidixic acid (NA), chloramphenicol (C), gentamicin (CN), trimethoprim/ sulfamethoxazole (SXT), colistin (CT), amoxicillin/clavulanic acid (AMC), and fosfomycin (FOT). 
All of the 144 isolates were resistant to at least one antimicrobial and exhibited the MDR phenotype. The MDR patterns of the isolates listed in Table-1 show a wide spectrum of antimicrobial resistance. We found a total of 29 MDR patterns reflecting an individual isolate's resistance to 3-9 antimicrobial classes. The MDR patterns with resistance to 9 classes of antimicrobials were observed only in $S$. Choleraesuis isolates. The most common MDR patterns among the serovars Choleraesuis and Rissen were R7-14 AMPAX-CAZ-CRO-CTX-NA-C-CN-TE (36, 25.00\%) followed by R7-18 AMP-AX-CRO-CTX-NA-CCN-TE $(15,10.42 \%)$.

\section{Discussion}

This is the first report linking serovars and antimicrobial resistance in Salmonella isolates obtained from clinical swine samples in Thailand. Our results identify the serovars originating from food-producing animals that require greater attention.

Overall, the serovar diversity among the clinical isolates was low, that is, only four Salmonella serovars were detected: Choleraesuis, monophasic Typhimurium, Typhimurium, and Rissen. These serovars were associated with various clinical samples or systemic infections of diseased swine. $S$. Choleraesuis is highly adapted to swine and usually causes swine paratyphoid with clinical manifestations of

Table-1: The MDR patterns of Salmonella serovars.

\begin{tabular}{|c|c|c|}
\hline Serovars & MDR patterns & No. of isolates $(\%)$ \\
\hline Choleraesuis $(n=96)$ & $\begin{array}{l}6 \text { classes } \\
\text { R6-23 AMP-AX-CAZ-CRO-CTX-NA-C-TE } \\
\text { R6-22 AMP-AX-CAZ-CRO-CTX-NA-CN-TE } \\
7 \text { classes } \\
\text { R7-14 AMP-AX-CAZ-CRO-CTX-NA-C-CN-TE } \\
\text { R7-18 AMP-AX-CRO-CTX-NA-C-CN-TE } \\
\text { R7-21 AMP-AX-CAZ-CRO-CTX-CIP-NA-CN-TE } \\
\text { R7-24 AMP-AX-CAZ-CTX-NA-CT-C-TE } \\
\text { R7-26 AMP-AX-CAZ-CRO-CTX-NA-CT-C-TE } \\
8 \text { classes } \\
\text { R8-6 AMP-AX-CAZ-CRO-CTX-NA-C-CN-TE-SXT } \\
\text { R8-15 AMP-AX-CAZ-CRO-CTX-NA-CT-C-CN-TE } \\
\text { R8-16 AMP-AX-CAZ-CRO-CTX-CIP-NA-C-CN-TE } \\
\text { R8-20 AMP-AX-CRO-CTX-NA-CT-C-CN-TE } \\
\text { 9 classes } \\
\text { R9-19 AMP-AX-CAZ-CRO-CTX-CIP-NA-C-CN-TE-SXT } \\
\text { R9-17 AMP-AX-CAZ-CRO-CTX-CIP-NA-CT-C-CN-TE } \\
\text { R9-25 AMP-AX-CRO-CTX-CIP-NA-C-CN-TE-SXT }\end{array}$ & $\begin{array}{l}21(14.58) \\
13(9.03) \\
8(5.56) \\
49(34.03) \\
30(20.83) \\
14(9.72) \\
2(1.39) \\
2(1.39) \\
1(0.69) \\
18(12.50) \\
8(5.56) \\
5(3.47) \\
4(2.78) \\
1(0.69) \\
8(5.56) \\
6(4.17) \\
1(0.69) \\
1(0.69)\end{array}$ \\
\hline $\begin{array}{l}\text { Monophasic Typhimurium } \\
(n=27)\end{array}$ & $\begin{array}{l}3 \text { classes } \\
\text { R3-3 AMP-AX-TE } \\
5 \text { classes } \\
\text { R5-2 AMP-AX-CT-C-TE } \\
\text { R5-4 AMP-AX-CRO-CTX-CN-TE } \\
\text { R5-8 AMP-AX-C-TE-SXT } \\
6 \text { classes } \\
\text { R6-1 AMP-AX-CAZ-CRO-CTX-C-CN-TE } \\
\text { R6-5 AMP-AX-CRO-CTX-CT-CN-TE } \\
\text { R6-10 AMP-AX-CRO-CTX-C-CN-TE } \\
\text { R6-7 AMP-AX-CT-C-TE-SXT } \\
\text { R6-9 AMP-AX-CTX-C-CN-TE } \\
\text { R6-12 AMP-AX-C-CN-TE-SXT } \\
7 \text { classes } \\
\text { R7-11 AMP-AX-CAZ-CRO-CTX-C-CN-TE-SXT } \\
\text { R7-13 AMP-AX-CT-C-CN-TE-SXT } \\
8 \text { classes } \\
\text { R8-6 AMP-AX-CAZ-CRO-CTX-NA-C-CN-TE-SXT }\end{array}$ & $\begin{array}{c}10(6.94) \\
10(6.94) \\
3(2.08) \\
1(0.69) \\
1(0.69) \\
1(0.69) \\
11(7.64) \\
3(2.08) \\
3(2.08) \\
2(1.39) \\
1(0.69) \\
1(0.69) \\
1(0.69) \\
2(1.39) \\
1(0.69) \\
1(0.69) \\
1(0.69) \\
1(0.69)\end{array}$ \\
\hline Typhimurium ( $n=13)$ & $\begin{array}{l}6 \text { classes } \\
\text { R6-1 AMP-AX-CAZ-CRO-CTX-C-CN-TE } \\
\text { R6-27 AMP-AX-CRO-CTX-CT-C-TE } \\
7 \text { classes } \\
\text { R7-29 AMP-AX-CAZ-CRO-CTX-CT-C-CN-TE } \\
\text { R7-28 AMP-AX-CRO-CTX-CT-C-CN-TE }\end{array}$ & $\begin{array}{l}10(6.94) \\
9(6.25) \\
1(0.69) \\
3(2.08) \\
2(1.39) \\
1(0.69)\end{array}$ \\
\hline Rissen $(n=8)$ & $\begin{array}{l}7 \text { classes } \\
\text { R7-14 AMP-AX-CAZ-CRO-CTX-NA-C-CN-TE } \\
\text { R7-18 AMP-AX-CRO-CTX-NA-C-CN-TE } \\
8 \text { classes } \\
\text { R8-16 AMP-AX-CAZ-CRO-CTX-CIP-NA-C-CN-TE }\end{array}$ & $\begin{array}{l}7(4.86) \\
6(4.17) \\
1(0.69) \\
1(0.69) \\
1(0.69)\end{array}$ \\
\hline
\end{tabular}

MDR=Multidrug resistant, $\mathrm{AMP}=$ Ampicillin, $\mathrm{AX}=$ Amoxicillin, $\mathrm{TE}=$ Tetracycline, $\mathrm{CTX}=$ Cefotaxime, $\mathrm{CRO}=$ Ceftriaxone, $\mathrm{C}=$ Chloramphenicol, $\mathrm{CN}=$ Gentamicin, $\mathrm{NA}=$ Nalidixic acid, $\mathrm{CAZ}=$ Ceftazidime, $\mathrm{CIP}=$ Ciprofloxacin, SXT=Trimethoprim/ sulfamethoxazole, $\mathrm{CT}=$ Colistin 
enterocolitis and septicemia. In humans, it frequently causes septicemic disease with little or no involvement of the gastrointestinal tract [4]. In Thailand, $S$. Choleraesuis swine infections are never reported in any official database because they cause mild symptoms that have never been associated with an outbreak. Therefore, it has never been microbiologically investigated. Thus, it is difficult to obtain a true picture of the occurrence of $S$. Choleraesuis infections in swine populations in Thailand.

At present, Salmonella 1,4,[5],12:i:- is considered a monophasic variant of $S$. Typhimurium that lacks the second-phase flagellar antigen. It has emerged as a major public health concern in multiple countries worldwide, including Thailand [20-24]. Pigs and pork products have often been implicated in outbreaks of monophasic $S$. Typhimurium. In this study, monophasic Typhimurium was more prevalent than $S$. Typhimurium and $S$. Rissen. Our results are not consistent with previous findings in Thailand suggesting that $S$. Rissen and $S$. Typhimurium are the most common serovars in swine [25-27]. The lack of agreement between studies may be due to the differences in study design and sample types.

AMP, C, and SXT are traditional first-line antimicrobial agents that have been used for treating Salmonella infections in humans since before the 1980s. By the late 1980s, MDR Salmonella had been observed in many countries [28-31]. Recently, extended-spectrum cephalosporins and fluoroquinolones have been suggested as alternative treatment options for MDR Salmonella infections in humans $[32,33]$. This study demonstrated that all clinical isolates belonging to serovars Choleraesuis, monophasic Typhimurium, Typhimurium, and Rissen were MDR, with resistances to 3-9 antimicrobial classes and exhibiting 29 distinct MDR patterns. The extremely high frequency of MDR among Salmonella serovars isolated from clinical swine samples is a remarkable observation that should raise concern. In Thailand, only one study has reported on antimicrobial use in swine farming [34], this study reported on the following antimicrobials: Penicillin, AX, cephalexin, TE, sulfadimethoxine, sulfamerazine, enrofloxacin, CIP, lincomycin, $\mathrm{CN}$, kanamycin, erythromycin, tiamulin, tylosin, and CT [34]. Their results are consistent with our findings regarding the most common MDR patterns. Collectively, these findings show that various MDR patterns in Salmonella serovars occur regardless of the level of overuse and misuse of antimicrobials during all stages of swine production.

This study also reports for the first time the high rate of resistance to third-generation cephalosporins (CAZ, CRO, and CTX) and reduced susceptibility to a fluoroquinolone (CIP) in $S$. Choleraesuis, $S$. Typhimurium, and $S$. Rissen obtained from clinical swine samples in Thailand. S. Choleraesuis, the most prevalent serovar in this study, is the second most common serovar implicated in human septicemia in Thailand $[25,35,36]$. In addition, recent studies have reported extremely high frequencies of $\mathrm{CRO}$ and fluoroquinolone resistance among invasive $S$. Choleraesuis isolated from bacteremic patients in Thailand $[25,35,36]$. Therefore, the chain of transmission and mechanism of resistance in MDR $S$. Choleraesuis should be further investigated to reduce the spread of resistance and its threat to human health in Thailand. It should be noted that although some Salmonella isolates were resistant to cephalosporins and fluoroquinolone in the present study, all isolates were susceptible to AMC acid and FOT.

This study was conducted as a passive surveillance on clinical samples that had been previously submitted for diagnostic purposes. Therefore, the results may not be representative of the general swine population in Thailand and potential biases exist regarding sample submissions. It is possible that our findings may have overestimated the true prevalence of MDR Salmonella among clinical isolates of swine populations in Thailand. Nevertheless, our approach provides an early warning for emerging pathogens and antimicrobial resistance threats in Thailand, and the results provide valuable information for managing Salmonella in swine.

\section{Conclusion}

Our results reveal the prevalence and resistance characteristics of Salmonella serovars in clinical swine samples for the first time in Thailand. Choleraesuis was the most predominant serovar followed by monophasic Typhimurium, Typhimurium, and Rissen. All isolates were MDR and collectively yielded 29 distinct MDR patterns. These results raise the alarm for the need to develop appropriate therapeutic treatments and call attention on the reasonable use of antimicrobials. The high rate of resistance to critical antimicrobials such as CAZ, CRO, and CTX and the reduced susceptibility to CIP among screened Salmonella serovars provide additional evidence on the possibility that such resistant strains may disseminate through the food supply chain, leading to therapeutic failures in food animals and humans. Finally, this study provides profiles of multidrug resistance among Salmonella serovars in clinically ill swine. These results provide useful information to improve strategies to control and treat Salmonella infections in swine populations in Thailand.

\section{Authors' Contributions}

SK designed the study, analyzed and interpreted the data, and drafted and revised the manuscript. SC performed the experiments and contributed reagents/ materials/analysis tools. DS and PN performed the experiments and collected the data. All authors have read and approved the final manuscript.

\section{Acknowledgments}

The authors are thankful to the Veterinary Diagnostic Laboratory, Faculty of Veterinary 
Medicine, Kasetsart University, for material support and the study participants for their kind cooperation. This study was supported by Huvepharma (Thailand) Ltd. (Grant No. VET59/HUVE01).

\section{Competing Interests}

The authors declare that research was conducted in the absence of any commercial or financial relationships that could be construed as a potential conflict of interest. No person from Huvepharma (Thailand) Ltd. was involved at any stage of the study.

\section{Publisher's Note}

Veterinary World remains neutral with regard to jurisdictional claims in published institutional affiliation.

\section{References}

1. Majowicz, S.E., Musto, J., Scallan, E., Angulo, F.J., Kirk, M., O'Brien, S.J., Jones, T.F., Fazil, A. and Hoekstra, R.M. (2010) The global burden of nontyphoidal Salmonella gastroenteritis. Clin. Infect. Dis., 50(6): 882-889.

2. Nair, S., Farzan, A., O'Sullivan,T.L. and Friendship, R.M. (2018) Time course of Salmonella shedding and antibody response in naturally infected pigs during grower-finisher stage. Can. J. Vet. Res., 82(2): 139-145.

3. Naberhaus, S.A., Krull, A.C., Arruda, B.L., Arruda, P., Sahin, O., Schwartz, K.J., Burrough, E.R., Magstadt, D.R., Ferreyra, F.M., Gatto, I.R.H., de Souza Almeida, H.M., Wang, C. and Kreuder, A.J. (2020) Pathogenicity and competitive fitness of Salmonella enterica serovar 4,[5],12:i: Compared to Salmonella Typhimurium and Salmonella Derby in Swine. Front. Vet. Sci., 6: 1-19.

4. Chiu, C.H., Su, L.H. and Chu, C. (2004) Salmonella enterica serotype choleraesuis: Epidemiology, pathogenesis, clinical disease, and treatment. Clin. Microbiol. Rev., 17(2): 311-322.

5. Farzan, A. and Friendship, RM. (2010) A clinical field trial to evaluate the efficacy of vaccination in controlling Salmonella infection and the association of Salmonellashedding and weight gain in pigs. Can. J. Vet. Res., 74(4): 258-263.

6. Campos, J., Mourão, J., Peixe, L. and Antunes, P. (2019) Non-typhoidal Salmonella in the pig production chain: A comprehensive analysis of its impact on human health. Pathogens, 8(1): 1-28.

7. Bonardi, S. (2017) Salmonella in the pork production chain and its impact on human health in the European Union. Epidemiol. Infect., 145(8): 1513-1526.

8. Lekagul, A., Tangcharoensathien, V. and Yeung, S. (2019) Patterns of antibiotic use in global pig production: A systematic review. Vet. Anim. Sci., 7: 1-12.

9. Kariuki, S., Gordon, M.A., Feasey, N. and Parry, C.M. (2016) Antimicrobial resistance and management of invasive Salmonella disease. Vaccine, 33(3): C21-C29.

10. Angulo, F.J. and Molbak, K. (2005) Human health consequences of antimicrobial drug-resistant Salmonella and other foodborne pathogens. Clin. Infect. Dis., 41(11): 1613-1620.

11. Phoon, YW., Chan, Y.Y.C. and Koh, T.H. (2015) Isolation of multidrug-resistant Salmonella in Singapore. Singapore Med. J., 56(8): e142-e144.

12. Jajere, S.M. (2019) A review of Salmonella enterica with particular focus on the pathogenicity and virulence factors, host specificity and adaptation and antimicrobial resistance including multidrug resistance. Vet. World, 12(4): 504-521.

13. Jiang, Z., Paudyal, N., Xu, Y., Deng, T., Li, F., Pan, H., Peng, X., He, Q. and Yue, M. (2019) Antibiotic resistance profiles of Salmonella recovered from finishing pigs and slaughter facilities in Henan, China. Front. Microbiol., 10: $1-9$.

14. Arguello, H., Sørensen, G., Carvajal, A., Baggesen, D.L., Rubio, P. and Pedersen, K. (2013) Prevalence, serotypes and resistance patterns of Salmonella in Danish pig production. Res. Vet. Sci., 95(2): 334-342.

15. Tadee, P., Kumpapong, K., Sinthuya, D., Yamsakul, P., Chokesaijawatee, N., Nuanualsuwan, S., Pornsukarom, S., Molla, B.Z., Gebreyes, W.A. and Patchanee, P. (2014) Distribution, quantitative load and characterization of Salmonella associated with swine farms in upper-Northern Thailand. J. Vet. Sci., 15(2): 327-334.

16. Nataro, J.P., Bopp, C.A., Fields, P.I., Kaper, J.B. and Strockbine, N.A. (2011) Escherichia, Shigella, Salmonella. In: Manual of Clinical Microbiology. $10^{\text {th }}$ ed. ASM Press, Washington, DC. p603-626.

17. Grimont, P.A.D. and Weill, F.X. (2007) Antigenic Formulae of the Salmonella Serovars. 9 $^{\text {th }}$ ed. WHO Collaborating Centre for Reference and Research on Salmonella, World Health Organization, Geneva.

18. Issenhuth-Jeanjean, S., Roggentin, P., Mikoleit, M., Guibourdenche, M., De Pinna, E., Nair, S., Fields, P.I. and Weill, F.X. (2014) Supplement 2008-2010 (No. 48) to the White-Kauffmann-Le Minor scheme. Res. Microbiol., 165(7): 526-530.

19. Clinical and Laboratory Standards Institute. (2017) Performance Standards for Antimicrobial Susceptibility Testing: Supplement M100-S23. 27 th $^{\text {th }}$ ed. Clinical and Laboratory Standards Institute, Wayne, PA.

20. Amavisit, P., Boonyawiwat, W. and Bangtrakulnont, A. (2005) Characterization of Salmonella enterica serovar Typhimurium and monophasic Salmonella serovar 1,4,[5],12:i: Isolates in Thailand. J. Clin. Microbiol., 43(6): 2736-2740.

21. Switt, A.I.M., Soyer, Y., Warnick, L.D. and Wiedmann, M. (2009) Emergence, distribution, and molecular and phenotypic characteristics of Salmonella enterica serotype 4,5,12:i:-. Foodborne Pathog. Dis., 6(4): 407-415.

22. Yang, X., Wu, Q., Zhang, J., Huang, J., Guo, W. and Cai S. (2015) Prevalence and characterization of monophasic Salmonella serovar 1,4,[5],12:i:-of food origin in China. PLoS One, 10(9): 1-10.

23. Proroga, Y.T.R., Mancusi, A., Peruzy, M.F., Carullo, M.R., Montone, A.M.I., Fulgione, A. and Capuano, F. (2019) Characterization of Salmonella Typhimurium and its monophasic variant 1,4, [5],12:i:- isolated from different sources. Folia Microbiol., 64(6): 711-718.

24. Arai, N., Sekizuka, T., Tamamura, Y., Tanaka, K., Barco, L., Izumiya, H., Kusumoto, M., Hinenoya, A., Yamasaki, S., Iwata, T., Watanabe, A., Kuroda, M., Uchida, I. and Akibaa, M. (2018) Phylogenetic characterization of Salmonella enterica serovar Typhimurium and its monophasic variant isolated from food animals in Japan revealed replacement of major epidemic clones in the last 4 decades. J. Clin. Microbiol., 56(5): 1-14.

25. Sirsichote, P., Bangtrakulnonth, A., Tianmanee, K., Unahalekhaka, A., Oulai, A., Chittaphithakchai, P., Kheowrod, W. and Hendriksen, R.S. (2010) Serotypes and antimicrobial resistance of Salmonella enterica spp in central Thailand, 2001-2006. Southeast Asian J. Trop. Med. Public Health, 41(6): 1405-1415.

26. Phongaran, D., Khang-Air, S. and Angkititrakul, S. (2019) Molecular epidemiology and antimicrobial resistance of Salmonella isolates from broilers and pigs in Thailand. Vet. World, 12(8): 1311-1318.

27. Patchanee, P., Tansiricharoenkul, K., Buawiratlert, T., Wiratsudakul, A., Angchokchatchawal, K., Yamsakul, P., Yano, T., Boonkhot, P., Rojanasatien, S. and Tadee, P. (2016) Salmonella in pork retail outlets and dissemination of its pulsotypes through pig production chain in Chiang Mai and surrounding areas, Thailand. Prev. Vet. Med., 130: 
99-105.

28. Harvey, R.R., Friedman, C.R., Crim, S.M., Judd, M., Barrett, K.A., Tolar, B., Folster, J.P., Griffin, P.M. and Brown, A.C. (2017) Epidemiology of Salmonella enterica serotype Dublin infections among humans, United States, 1968-2013. Emerg. Infect. Dis., 23(9): 1493-1501.

29. Mangat, C.S., Bekal, S., Avery, B.P., Côté, G., Daignault, D., Doualla-Bell, F., Finley, R., Lefebvre, B., Bharat, A., Parmley, E.J., Reid-Smith, R.J., Longtin, J., Irwin R.J. and Mulvey, M.R. (2019) Genomic investigation of the emergence of invasive multidrug-resistant Salmonella enterica serovar Dublin in humans and animals in Canada. Antimicrob. Agents Chemother., 63(6): 1-18.

30. Hawkey, J., Le Hello, S., Doublet, B., Granier, S.A., Hendriksen, R.S., Fricke, W.F., Ceyssens, P.J., Gomart, C., Billman-Jacobe, H., Holt, K.E. and Weill, F.X. Global phylogenomics of multidrug-resistant Salmonella enterica serotype kentucky ST198. Microb. Genom., 5(7): e000269.

31. Browne, A.J., Hamadani, B.H.K., Kumaran, E.A.P., Rao, P., Longbottom, J., Harriss, E., Moore, C.E., Dunachie, S., Basnyat, B., Baker, S., Lopez, A.D., Day, N.P.J., Hay, S.I. and Dolecek, C. (2020) Drug-resistant enteric fever worldwide, 1990 to 2018: A systematic review and meta-analysis. BMC Med., 18(1): 1-22.

32. Crump, J.A., Sjölund-Karlsson, M., Gordon, M.A. and
Parry, C.M. (2015) Epidemiology, clinical presentation, laboratory diagnosis, antimicrobial resistance, and antimicrobial management of invasive Salmonella infections. Clin. Microbiol. Rev., 28(4): 901-937.

33. Shane, A.L., Mody, R.K., Crump, J.A., Tarr, P.I., Steiner, T.S., Kotloff, K., Langley, J.M., Wanke, C., Warren, C.A., Cheng, A.C., Cantey, J. and Pickering, L.K. (2017) 2017 infectious diseases society of America clinical practice guidelines for the diagnosis and management of infectious diarrhea. Clin. Infect. Dis., 65(12): 1963-1973.

34. Love, D.C., Tharavichitkul, P., Arjkumpa, O. and Imanishi, M. (2015) Antimicrobial use and multidrug-resistant Salmonella spp., Escherichia coli, and Enterococcus faecalis in Swine from Northern Thailand. Thai J. Vet. Med., 45(1): 43-53.

35. Kulwichit, W., Chatsuwan, T., Unhasuta, C., Pulsrikarn, C., Bangtrakulnonth, A. and Chongthaleong, A. (2007) Drugresistant nontyphoidal Salmonella bacteremia, Thailand. Emerg. Infect. Dis., 13(3): 501-502.

36. Luk-in, S., Chatsuwan, T., Pulsrikarn, C., Bangtrakulnonth, A., Rirerm, U. and Kulwichit, W. (2018) High prevalence of ceftriaxone resistance among invasive Salmonella enterica serotype Choleraesuis isolates in Thailand: The emergence and increase of CTX-M-55 in ciprofloxacin-resistant $S$. choleraesuis isolates. Int. J. Med. Microbiol., 308(4): 447-453.

\section{$* * * * * * * *$}

